\title{
THE INFLUENCE OF SURFACE IRREGULARITIES UPON THE VAN DER WAALS FORCES BETWEEN MACROSCOPIC BODIES
}

\author{
J.L.M.J. VAN BREE, J.A. POULIS and B.J. VERHAAR \\ Afdeling Natuurkunde der Technische Hogeschool, \\ Eindhoven, The Netherlands \\ and \\ K. SCHRAM \\ Instituut voor Theoretische Fysica der Rijksuniversiteit, \\ Utrecht, The Netherlands
}

Received 2 July 1974

\section{Synopsis}

An expression is derived for the Van der Waals force between two semi-infinite bodies with small surface irregularities. Calculations are given both for the plane-plane and the plane-sphere configuration. The value of the correction from the surface irregularitics upon the Van der Waals force is shown to amount easily to $10-50 \%$.

1. Introduction. During the last decades the subject of Van der Waals forces between macroscopic bodies has drawn much attention both from the theoretical and experimental point of view. For technical reasons one usually measures attractive forces which act between two semi-infinite bodies facing each other with a plane and a spherical surface, respectively. To make comparison possible between theory and experiment the theories also are concentrated on these configurations. The relevance of the comparison however is somewhat influenced by the fact that the theories deal with ideal surfaces, while experiments are carried out with real surfaces, which are not free from irregularities.

The present paper will discuss the effect of irregularities on the comparison between theory and experiment. We shall discuss both a configuration of two nonideal bodies facing each other with plane-parallel surfaces and a configuration of a spherical surface facing a plane one.

2. Plane-parallel surfaces. The two plane surfaces are taken to be mutually parallel at a distance $d$. We shall start the calculation with one surface being ideally flat while the other having a roughness characterised by $\left(\overline{\zeta^{2}}\right)^{\frac{1}{2}}$, which stands for 
the r.m.s. of the deviations $\zeta$ from an ideal flat plane defined such that the average deviation is zero $(\bar{\zeta}=0$, see fig. 1$)$.

Starting with the nonretarded situation we shall use the well-known expression for the interaction energy $U_{0}(z)$ of a single harmonic oscillator with a semi-infinite body consisting of the same kind of oscillators

$$
U_{0}(z)=-\frac{1}{8} \pi \hbar \omega_{0} \varrho \alpha^{2}\left(1 / z^{3}\right),
$$

in which $z$ is the distance of the oscillator to the plane surface, $\varrho$ the oscillator density, $\alpha$ the static polarizability and $\omega_{0}$ stands for the resonance frequency of the oscillator. In this expression the forces between oscillators are assumed to be

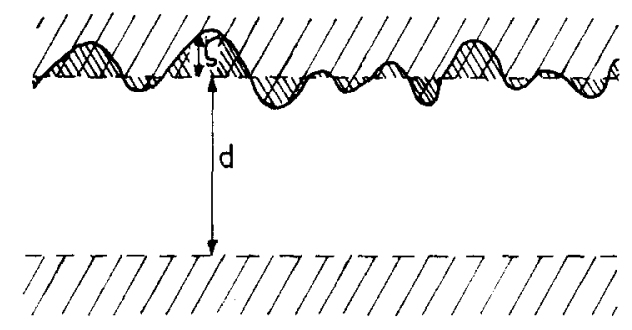

Fig. 1. Van der Waals force in the plane-plane configuration.

additive and the limitation to the nonretarded situation can be expressed by $\omega_{0} z \ll c$. The correction to the interaction energy due to the irregularities is considered to be made up of contributions from the volume elements $\mathrm{d} x \mathrm{~d} y \mathrm{~d} z$ near the irregular surface, which are to be added or substracted when $\zeta$ is negative or positive, respectively. For each of these contributions we use $\mathrm{d} U(z)=U_{0}(z)$ $\times \varrho \mathrm{d} x \mathrm{~d} y \mathrm{~d} z$. The contribution $U_{\mathrm{c}}(d) / L^{2}$ of the irregularities to the interaction energy per unit area is given by

$$
U_{\mathrm{c}}(d) / L^{2}=-\frac{1}{8} \pi \hbar \omega_{0} \varrho^{2} \alpha^{2}\left(1 / L^{2}\right) \int_{-L / 2}^{+L / 2} \mathrm{~d} x \int_{-L / 2}^{+L / 2} \mathrm{~d} y \int_{d+\zeta(x, y)}^{d}\left(1 / z^{3}\right) \mathrm{d} z,
$$

in which $L$ stands for the dimension of the surface area considered and $\zeta(x, y)$ represents the deviation as a function of the position $(x, y)$ on the ideal plane. Carrying out the integration over $z$ and using the approximation $\zeta \ll d$, eq. (2) becomes

$$
U_{\mathrm{c}}(d) / L^{2}=-\frac{3}{16} \pi \hbar \omega_{0} \varrho^{2} \alpha^{2}\left(1 / d^{4}\right) \overline{(\zeta(x, y))^{2}},
$$

where the averaging is performed over the surface area $L$. Irregularities on the opposite surface can simply be taken into account by repeating the procedure 
sketched above. By differentiating we then get for the contribution to the Van der Waals force per unit area due to the irregularities

$$
F_{\mathrm{c}}(d) / L^{2}=\frac{3}{4} \pi \hbar \omega_{0} Q^{2} \alpha^{2}\left(1 / d^{5}\right)\left(\overline{\zeta_{1}^{2}}+\overline{\zeta_{2}^{2}}\right)
$$

where $\zeta_{1}(x, y)$ and $\zeta_{2}(x, y)$ represent the irregularities on the two surfaces.

This expression for $F_{\mathrm{c}}$ is to be compared with an expression for the unperturbed Van der Waals force between the semi-infinite half-spaces. As an example we shall use the expression for the unperturbed force obtained by summing the pair interactions over the two media ${ }^{2}$ )

$$
F(d) / L^{2}=\frac{1}{8} \pi \hbar \omega_{0} \varrho^{2} \alpha^{2}\left(1 / d^{3}\right) .
$$

Comparing this result to (4) we get

$$
F_{\mathrm{c}}(d) / F(d)=6\left(1 / d^{2}\right)\left(\overline{\zeta_{1}^{2}}+\overline{\zeta_{2}^{2}}\right) .
$$

For the retarded case where $\omega_{0} d \gg c$ a similar calculation using the additive part of the equation of Nijboer and Renne ${ }^{6,7}$ ) for the interaction between a single oscillator and a semi-infinite medium we get

$$
F_{\mathrm{c}}(d) / L^{2}=\frac{23}{4} \hbar c \varrho^{2} \alpha^{2}\left(1 / d^{6}\right)\left(\overline{\zeta_{1}^{2}}+\overline{\zeta_{2}^{2}}\right) .
$$

Comparing this with the expression for the unperturbed force ${ }^{6,7}$ ) we get

$$
F_{\mathrm{c}}(d) / F(d)=10\left(1 / d^{2}\right)\left(\overline{\zeta_{1}^{2}}+\overline{\zeta_{2}^{2}}\right) .
$$

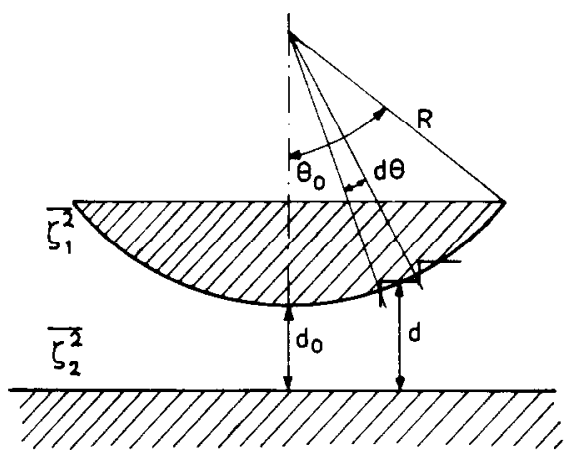

Fig. 2. The plane-sphere configuration.

3. Plane-sphere configuration. Actual measurements, however, are usually not performed with bodies facing each other with plane surfaces but with a body with a plane surface facing a spherical body. We shall start the discussion of the influence of irregularities in this case by considering the nonretarded situation. In 
order to make possible the application of eq. (3) we shall replace the spherical surface by a combination of plane rings as shown in fig. 2 . The surface area referred to as $L^{2}$ in eq. (4) we therefore replace by $2 \pi R^{2} \sin \theta \mathrm{d} \theta$. When $d_{0}$ is the shortest distance between plane and sphere, the distance between one of the rings and the plane is given by $d=d_{0}+R(1-\cos \theta)$. When $\bar{\zeta}_{2}^{2}$ and $\bar{\zeta}_{1}^{2}$ again represent the irregularities of the surface of the sphere and plane, respectively, get by summation over the rings and using the restriction $R\left(1-\cos \theta_{0}\right) \gg d_{0}$ (which is commonly satisfied in practice):

$$
F_{\mathrm{c}}\left(d_{0}\right)=\frac{3}{8} \pi^{2} \hbar \omega_{0} \varrho^{2} \alpha^{2} R\left(1 / d_{0}^{4}\right)\left(\overline{\zeta_{1}^{2}}+\overline{\zeta_{2}^{2}}\right)
$$

With the expression of the unperturbed force ${ }^{3}$ ) we get

$$
F_{\mathrm{c}}\left(d_{0}\right) / F\left(d_{0}\right)=3\left(1 / d_{0}^{2}\right)\left(\overline{\zeta_{1}^{2}}+\overline{\zeta_{2}^{2}}\right)
$$

The same procedure in the retarded case leads to

$$
F_{\mathrm{c}}\left(d_{0}\right)=\frac{23}{10} \pi \hbar c \varrho^{2} \alpha^{2} R\left(1 / d_{0}^{5}\right)\left(\overline{\zeta_{1}^{2}}+\overline{\zeta_{2}^{2}}\right),
$$

which together with the unperturbed force results in

$$
F_{\mathrm{c}}\left(d_{0}\right) / F\left(d_{0}\right)=6\left(1 / d_{\mathrm{o}}^{2}\right)\left(\overline{\zeta_{1}^{2}}+\overline{\zeta_{2}^{2}}\right) .
$$

4. Conclusion. The contribution of the irregularities to the Van der Waals force is most relevant for the nonretarded situation. For example in certain measurements by Tabor and Winterton ${ }^{4}$ ) and by Israelachvili and Tabor ${ }^{5}$ ) in which the distance is smaller than $10 \mathrm{~nm}$, it follows from eq. (6) that surface irregularities as small as $2 \mathrm{~nm}$ give rise to an effect of at least 50 percent in the plane-plane and 25 percent in the plane-sphere situation. In the retarded case for distances greater than $20 \mathrm{~nm}$ similar irregularitics give according to cq. (8) rise to a contribution smaller than 15 percent in the plane-plane and 10 percent in the plane-sphere situation.

\section{REFERENCES}

1) London, F., Z. phys. Chemie (B) 11 (1930) 222.

2) De Boer, J.H., Trans. Faraday Soc. 32 (1936) 10.

3) Hamaker, H.C., Physica 4 (1937) 1058.

4) Tabor, D. and Winterton, R.H.S., Proc. Roy. Soc. A132 (1969) 435.

5) Israelachvili, J. N. and Tabor, D., Proc. Roy. Soc. A331 (1972) 19.

6) Renne, M.J., Physica 56 (1971) 125.

7) Nijboer, B. R. A. and Renne, M. J., Chem. Phys. Letters 2 (1968) 35. 